Proyecciones Journal of Mathematics

Vol. 33, No 1, pp. 123-132, March 2014.

Universidad Católica del Norte

Antofagasta - Chile

\title{
Birrepresentations in a locally nilpotent variety
}

\author{
Manuel Arenas* \\ and \\ Alicia Labra ${ }^{\dagger}$ \\ Universidad de Chile, Chile \\ Received : June 2013. Accepted : November 2013
}

\begin{abstract}
It is known that commutative algebras satisfying the identity of degree four $((y x) x) x+\gamma y((x x) x)=0$, with $\gamma$ in the field and $\gamma \neq-1$ are locally nilpotent. In this paper we study the birrepresentations of an algebra $A$ that belongs to a variety $\mathcal{V}$ of locally nilpotent algebras. We prove that if the split null extension of a birrepresentation of an algebra $A \in \mathcal{V}$ by a vector space $M$ is locally nilpotent, then it is trivial or reducible. As corollaries we get that if $A$ is finitely generated, then every birrepresentation is trivial or reducible and that every finite-dimensional birrepresentation is equivalent to a birrepresentation consisting of strictly upper triangular matrices. We also prove that the multiplicative universal envelope of a finitely generated algebra in $\mathcal{V}$ is nilpotent, therefore it is finite-dimensional.
\end{abstract}

${ }^{*}$ Supported by Fondecyt 1120844 .

${ }^{\dagger}$ Supported by Fondecyt 1120844. 


\section{Introduction}

Let $A$ be a commutative algebra over a field $K$, of characteristic $\neq 2$, satisfying the identity of degree four

$$
((y x) x) x+\gamma y((x x) x)=0 .
$$

Remark 1. It is immediate that every commutative algebra $A$ satisfying (1.1) with $\gamma \neq-1$, satisfies the identity

$$
((x x) x) x=0 .
$$

A very important unsolved problem in non-associative algebra is the one known as Albert's problem. This problem consists in to find out whether every finitely dimensional commutative power-associative algebra is solvable. The usual approaches to solve this problem consist in to consider specific values of some parameters as the dimension or the nilindex of the algebra. A different approach consists in to assume that the algebra satisfies other additional identities.

With this aim and considering remark 1, Correa, Hentzel and Labra [CHL] studied commutative algebras satisfying (1.1). In that paper they proved that any finitely generated algebra satisfying (1.1) with $\gamma \in\{0,1\}$ is nilpotent. Later Behn, Elduque and Labra in [BEL] generalized this result by proving that if $\gamma \neq-1$, then any such algebra is locally nilpotent.

Example 1. Let $A$ be a commutative real algebra with basis $\left\{x_{1}, x_{2}, x_{3}, z\right\}$ with the following multiplication table:

\begin{tabular}{c|cccc} 
& $x_{1}$ & $x_{2}$ & $x_{3}$ & $z$ \\
\hline$x_{1}$ & $x_{2}$ & $x_{3}$ & 0 & 0 \\
$x_{2}$ & $x_{3}$ & $x_{3}$ & 0 & $x_{3}$ \\
$x_{3}$ & 0 & 0 & 0 & 0 \\
$z$ & 0 & $x_{3}$ & 0 & $x_{2}+x_{3}$
\end{tabular}

We observe that for every $a, b, c, d \in A$, we have that $a b \in \mathbf{R} x_{2}+\mathbf{R} x_{3},(a b) c \in \mathbf{R} x_{3}$ and $((a b) c) d=0$. As a consequence we have that $((y x) x) x=y((x x) x)=0$. Then $A$ satisfies (1.1) for every $\gamma \in \mathbf{R}$.

\section{Representations and birrepresentations}

Let $A$ be an algebra over a field $K$ which belongs to a variety $\mathcal{V}$. Let $M$ be a vector space over $K$. As in Eilenberg [Eil], we define a birrepresentation 
of $A$ in $M$ as a linear function $(\rho, \lambda): A \longrightarrow \operatorname{End}(M) \times \operatorname{End}(M)$. We say that the birrepresentation $(\rho, \lambda)$ is a birrepresentation in the variety $\mathcal{V}$ if the space $S=A \oplus M$ endowed with the multiplication given by

$$
(x+m)(y+n)=x y+\rho(y)(m)+\lambda(x)(n)
$$

for every $x, y$ in $A$ and $m, n$ in $M$, is an algebra in the variety $\mathcal{V}$. The space $M$ becomes a bimodule over $A$. The algebra $S$ is called the split null extension of $A$ by the bimodule $M$ given by $(\rho, \lambda)$. If the variety $\mathcal{V}$ is contained in the variety of commutative algebras, then in the definition above $\rho=\lambda$, we call this map $\mu$ and we talk about $\mu$ instead of $(\rho, \lambda)$. In this case $\mu$ is called a representation and $M$ becomes a module over $A$.

Lemma 1. Let $A$ be a commutative algebra over a field $K$ with characteristic $\neq 2$, satisfying (1.1) and $M$ be a linear space over $K$. Then, a linear map $\mu: A \rightarrow \operatorname{End}(M)$ is a representation of $A$ if and only if for every $x$ and $y$ in $A$ the following identities are satisfied:

$$
\mu((y x) x)+\mu(x) \mu(y x)+\mu(x)^{2} \mu(y)+\gamma\left[\mu(y) \mu\left(x^{2}\right)+2 \mu(y) \mu(x)^{2}\right]=0 .
$$

$$
\mu(x)^{3}+\gamma \mu\left(x^{3}\right)=0 .
$$

Proof. For all $x, y$ in $A$ and $m, n$ in $M$ we have that $S=A \oplus M$ satisfies identity (1.1), that is:

$$
(((y+n)(x+m))(x+m))(x+m)+\gamma(y+n)(x+m)^{3}=0
$$

holds in $S$. Therefore, for every, $x, y \in A, m, n \in M$ we have:

$$
\begin{gathered}
((y x) x) x+\mu((x y) x)(m)+\mu(x) \mu(x y)(m)+\mu(x)^{2} \mu(y)(m)+\mu(x)^{3}(n) \\
+\gamma\left[y x^{3}+\left[\mu(y) \mu\left(x^{2}\right)+2 \mu(y) \mu(x)^{2}\right](m)+\mu\left(x^{3}\right)(n)\right]=0 .
\end{gathered}
$$

Therefore, $\mu$ is a representation of $A$ if and only if the identities (2.1) and (2.2) hold. 
Example 2. Let us consider the algebra $A$ of Example 1. Let $M$ be a three dimensional space over $K$ and define $\mu: A \longrightarrow \operatorname{End}(M)$ such that the matrix of $\mu(x)$ in the a fixed basis is

$$
\mu(x)=\left(\begin{array}{ccc}
0 & \alpha_{1} & \alpha_{4} \\
0 & 0 & \alpha_{1} \\
0 & 0 & 0
\end{array}\right)
$$

for every $x=\alpha_{1} x_{1}+\alpha_{2} x_{2}+\alpha_{3} x_{3}+\alpha_{4} z \in A$. We are going to prove that $\mu$ is a representation of $A$.

If we consider $x$ as above and $y=\beta_{1} x_{1}+\beta_{2} x_{2}+\beta_{3} x_{3}+\beta_{4} z$, we have that

$$
y x=\left(\alpha_{1} \beta_{1}+\alpha_{4} \beta_{4}\right) x_{2}+\left(\alpha_{1} \beta_{2}+\alpha_{2} \beta_{1}+\alpha_{2} \beta_{2}+\alpha_{2} \beta_{4}+\alpha_{4} \beta_{2}+\alpha_{4} \beta_{4}\right) x_{3}
$$

and

$$
(y x) x=\left(\alpha_{1}^{2} \beta_{1}+\alpha_{1} \beta_{4} \alpha_{4}+\alpha_{1} \beta_{1} \alpha_{2}+\alpha_{4} \beta_{4} \alpha_{2}+\alpha_{1} \beta_{1} \alpha_{4}+\alpha_{4}^{2} \beta_{4}\right) x_{3}
$$

Therefore, straightforward computations gives $\mu((y x) x)=0, \quad \mu(y x)=$ $0, \mu\left(x^{2}\right)=0, \mu\left(x^{3}\right)=0$,

$$
\mu(x)^{2}=\left(\begin{array}{ccc}
0 & 0 & \alpha_{1}^{2} \\
0 & 0 & 0 \\
0 & 0 & 0
\end{array}\right),
$$

and $\mu(x)^{3}=0$. Thus $\mu(x)^{3}+\gamma \mu\left(x^{3}\right)=0$ for every $x$ and

$$
\begin{gathered}
\mu((y x) x)+\mu(x) \mu(y x)+\mu(x)^{2} \mu(y)+\gamma\left[\mu(y) \mu\left(x^{2}\right)+2 \mu(y) \mu(x)^{2}\right]= \\
\left(\begin{array}{ccc}
0 & 0 & \alpha_{1}^{2} \\
0 & 0 & 0 \\
0 & 0 & 0
\end{array}\right)\left(\begin{array}{ccc}
0 & \beta_{1} & \beta_{4} \\
0 & 0 & \beta_{1} \\
0 & 0 & 0
\end{array}\right)+2 \gamma\left(\begin{array}{ccc}
0 & \beta_{1} & \beta_{4} \\
0 & 0 & \beta_{1} \\
0 & 0 & 0
\end{array}\right)\left(\begin{array}{ccc}
0 & 0 & \alpha_{1}^{2} \\
0 & 0 & 0 \\
0 & 0 & 0
\end{array}\right)=0 .
\end{gathered}
$$

Hence Lemma 1 proves that $\mu$ is a representation of $A$.

\section{Birrepresentations in an arbitrary locally nilpotent variety}

Since it was proved in [BEL] that the variety of commutative algebras satisfying (1.1) with $\gamma \neq-1$ is locally nilpotent, in order to study representations of algebras in this particular family of varieties, we are going to 
give some general results for birrepresentatios of algebras in an arbitrary locally nilpotent variety.

We say that a birrepresentation $(\rho, \lambda)$ of $A$ is irreducible if $M \neq 0$ and there is no proper non-zero subspace of $M$ which is invariant under all the transformations $\rho(a), \lambda(a) \in \operatorname{End}(M)$. Clearly, in Example 2, the representation described is not irreducible.

Remark 2. In what follows we have some results for finitely generated algebras in a locally nilpotent variety. Nevertheless, it is important to say that, if an algebra $A$ is nilpotent and finitely generated, it has to be finitedimensional. Let $G$ be a set of generators of $A$ and $V$ is the vector space spanned by $G$. We have that $A=\sum_{n=1}^{\infty} V^{n}$ hence, when $A$ is nilpotent the sum is finite and when $G$ is finite $V^{n}$ has finite dimension for every $n$, and the result follows.

Lemma 2. Let $A$ be a finitely generated algebra. Let $M \neq\{0\}$ be a $K$ vector space and $(\rho, \lambda)$ is an birrepresentation of $A$. We have that if $M$ is irreducible or finitely generated, then the split null extension $S=A \oplus M$ is finitely generated.

Proof. Let be $B=\langle\rho(a), \lambda(a) \mid a \in A\rangle$ the subalgebra of $\operatorname{End}(M)$ generated by $\{\rho(a), \lambda(a) \mid a \in A\}$. Let be $x_{1}, \ldots, x_{n}$ be a set of generators of $A$. Suppose $M$ irreducible. Let $m$ be a non zero element of $M$. We are going to prove that $S=\left\langle x_{1}, \ldots, x_{n}, m\right\rangle$. It is obvious that the subspace $B m$ of $M$ is a submodule of $M$. Since $M$ is irreducible $B m=\{0\} \vee B m=M$. If $B m=\{0\}$, then $M=K m$ and $S=A \oplus K m=\left\langle x_{1}, \ldots, x_{n}, m\right\rangle$. Suppose now that $B m=M$. For every $x \in S$ we have $x=a+n$ with $a \in A \subseteq\left\langle x_{1}, \ldots, x_{n}, m\right\rangle$ and $n \in B m$. This implies that $n$ is a sum of products of $m$ by elements in $A$, so $n \in\left\langle x_{1}, \ldots, x_{n}, m\right\rangle$. Finally we get that $S=\left\langle x_{1}, \ldots, x_{n}, m\right\rangle$. If $M$ is finitely generated there are $m_{1}, \ldots m_{k} \in M$ such that every element $n \in M$ is a sum of products of $m_{i}$ by elements of $A$, thus $n \in\left\langle x_{1}, \ldots x_{n}, m_{1}, \ldots, m_{k}\right\rangle$. Finally we get that $S=\left\langle x_{1}, \ldots, x_{n}, m_{1}, \ldots, m_{k}\right\rangle$.

Corollary 1. Let $A$ be an algebra in $\mathcal{V}$. Let $(\rho, \lambda)$ be the birrepresentation of $A$ into the vector space $M$, such that the split null extension $S=A \oplus M$ is finitely generated. Then $B=\langle\lambda(a), \rho(a) \mid a \in A\rangle$ is a nilpotent algebra.

Proof. Since $\mathcal{V}$ is locally nilpotent and $S$ is finitely generated, we have that $S$ it is nilpotent. A simple induction proves that $B^{k} M \subseteq S^{k+1}$ for every natural $k$, therefore $B$ is nilpotent. 
Theorem 1. Let $A$ be an arbitrary algebra and let $(\rho, \lambda)$ a birrepresentation of $A$ by a vector space $M$ such that the split null extension $S=A \oplus M$ is locally nilpotent. Therefore $M$ is trivial or reducible.

Proof. Let $B$ the subalgebra of $\operatorname{End}(M)$ generated by $\{\lambda(a), \rho(a) \mid a \in$ $A$ \}. Suppose $M$ is non trivial. That means that there is an element $m \in M$ such that the submodule $B m$ is not $\{0\}$.

If $B m=M$, then there exists $b \in B$, such that $m=b m$. Since $b \in B$, there is a finite set $F \subseteq A$ such that $b$ is a sum of products of endomorphisms $\xi(a)$ with $\xi \in\{\rho, \lambda\}$ and $a \in F$.

Let $T$ be the subalgebra of $S$ generated by the finite set $F \cup\{m\}$. Since $S$ is locally nilpotent, we have that $T$ is nilpotent. Since $m=b m$ a simple induction proves that $b^{k} m=m$ for every natural $k$. On the other hand, since $b m \in T^{2}$ a simple induction proves $b^{k} m \in T^{k+1}$ for every natural $k$. We conclude that $m=0$ which contradicts the fact that $B m=M$ and $M$ is not trivial. We conclude that $B m \notin\{M,\{0\}\}$, so $M$ is reducible.

The three corollaries below are valid for every variety $\mathcal{V}$ of locally nilpotent non necessarily commutative algebras. In particular are valid for the variety of commutative algebras satisfying (1.1) with $\gamma \neq-1$.

Corollary 2. Let $A$ be a finitely generated algebra in $\mathcal{V}$. Then every birrepresentation of $A$, is trivial or reducible.

Proof. Let $(\rho, \lambda)$ be the birrepresentation of $A$ into the vector space $M$ and suppose it is irreducible. By Lemma 2 the split null extension $S$ is finitely generated, so Theorem 1 implies that $M$ is trivial.

Corollary 3. Let $A$ be a finitely generated algebra in $\mathcal{V}$ and let $(\rho, \lambda)$ be a birrepresentation of $A$ into a vector space $M$ which is finitely generated as an $A$-module. Therefore there exists a basis of $M$ such that the matrix of $\rho(x)$ and $\lambda(x)$ in that basis is strictly upper triangular for every $x \in A$.

Proof. Since $A$ is finitely generated and $M$ is finitely generated, Lemma 2 implies that $S$ is finitely generated and by remark $2 S$ has finite dimension, so $M$ has finite dimension. At this point Corollary 1 lead us to conclude that $B=\langle\lambda(a), \rho(a) \mid a \in A\rangle$ is a nilpotent associative algebra. The result follows form the fact that $M$ is a finitely dimensional module in the associative sense over the nilpotent associative algebra $B$. 


\section{The multiplicative universal envelope}

Let $\mathcal{V}$ be a variety of algebra and let $A$ be an algebra in $\mathcal{V}$. The multiplicative universal envelope of $A$ is the unique associative algebra (up to isomorphism) $\mathcal{M}(A)$, endowed with a linear function $(R, L): A \longrightarrow$ $\mathcal{M}(A) \times \mathcal{M}(A)$, such that for every birrepresentation $(\rho, \lambda)$ in $\mathcal{V}$ from $A$ into $\operatorname{End}(M) \times \operatorname{End}(M)$ for a vector space $M$, there is a unique homomorphism $\phi: \mathcal{M}(A) \longrightarrow \operatorname{End}(M)$ of associative algebras such that $\phi \circ R=\rho$ and $\phi \circ L=\lambda$.

Let $K\{X\}$ and $\mathcal{A S S}\{X\}$ respectively be the free algebra and the associative free algebra generated by a set of symbols $X=\left\{x_{i}\right\}_{i=1}^{\infty}$. Let be $f\left(x_{1}, \ldots, x_{n}\right) \in K\{X\}$ one of the defining identities of a variety $\mathcal{V}$. There are some elements $\left(f_{i}\right)_{i=1}^{n}$ of $K\{X\}$ such that for every $A \in \mathcal{V}$ and every $A$ module $M$ we have that $f\left(a_{1}+m_{1}, \ldots, a_{n}+m_{n}\right)=\sum_{i=1}^{k} f_{i}\left(a_{1}, \ldots, a_{n}, m_{i}\right)$ in $A \oplus M$ for every $a_{1}, \ldots a_{n} \in A, m_{1}, \ldots, m_{n} \in M$. Note that for every element $h \in K\{X\}$ if we evaluate $h\left(b_{1}, \ldots, b_{r}\right)$ where at least two of those elements are in $M$ the result is always zero.

For every index $i$ and every list $\left(a_{1} \ldots a_{n}\right)$ of elements of $A$, the function $m \longrightarrow f_{i}\left(a_{1}, \ldots, a_{n}, m\right)$ is an element $P_{i}$ of $\operatorname{End}(M)$.

It is possible to find elements $g_{i}$ of $\mathcal{A S S}\{X\}$ such that the map $\left(a_{1}, \ldots, a_{n}\right)$ $\longrightarrow P_{i}$ has the form

$$
P_{i}=g_{i}\left(\rho\left(y_{1}\right), \ldots, \rho\left(y_{r}\right), \lambda\left(y_{1}\right), \ldots, \lambda\left(y_{r}\right)\right),
$$

where for every $j$, the element $y_{j}$ is obtained by evaluating a polynomial identity of $K\{X\}$ in the tuple $\left(a_{1}, \ldots, a_{n}\right)$.

Therefore for every $A$ in $\mathcal{V}$ and every birrepresentation $(\rho, \lambda)$ is a birrepresentation in $\mathcal{V}$ if and only if we have that $g_{i}\left(\rho\left(y_{1}\right), \ldots, \rho\left(y_{r}\right), \lambda\left(y_{1}\right), \ldots, \lambda\left(y_{r}\right)\right)$ $=0$ for every $i$ and for every $n$-tuple $\left(a_{1}, \ldots, a_{n}\right)$ of elements of $A$.

Of course all this can be generalized to a variety defined by more that one identity, we only get a larger set of elements $g_{i}$ in $\mathcal{A S S}(X)$. We call these identities the birrepresentation defining identities of the variety $\mathcal{V}$.

As an example, we can see that if $A$ is an associative algebra and $(\rho, \lambda)$ is a birrepresentation, it is an associative birrepresentation if and only if $\rho(x) \rho(y)=\rho(x y)$ and $\lambda(x) \lambda(y)=\lambda(x y)$. So, if we define the associative polynomials in five variables $g_{1}(a, b, c, d, e, f)=a b-c$ and $g_{2}(a, b, c, d, e, f)=d-e f$, we have that $(\rho, \lambda)$ is an associative birrepresentation if and only if

$$
g_{i}(\rho(x), \rho(y), \rho(x y), \lambda(x), \lambda(y), \lambda(x y))=0,
$$


for $i \in\{1,2\}$. Therefore the set of birrepresentation defining identities is $\left\{g_{1}, g_{2}\right\}$.

It is immediate that we can avoid the terms that do not appear in the expression and write $g_{1}(a, b, c)=a b-c$ and $g_{2}(d, e, f)=d-e f$ for short.

In the variety defined by the commutativity and identity (1.1), since we have the identities (2.1) and (2.2), the birrepresentation defining identities are $g_{1}(\rho(x), \lambda(x))=0, g_{2}\left(\rho(x), \rho(y), \rho\left(x^{2}\right), \rho(y x), \rho((y x) x)\right)=0$, and $g_{3}\left(\rho(x), \rho\left(x^{3}\right)\right)=0$ where

$$
\begin{gathered}
g_{1}(a, b)=a-b \\
g_{2}(a, b, c, d, e)=e+a d+a^{2} b+\gamma\left[b c+2 b a^{2}\right] \\
g_{3}(a, b)=a^{3}+\gamma b
\end{gathered}
$$

Now we proceed to build the multiplicative universal envelope of $A$ as in [Um]. Let $R(A)$ and $L(A)$ be two copies of $A$ as a vector space. Let $\mathcal{M}(A)$ be the free associative algebra generated by the vector space $L(A) \oplus R(A)$ with the relations $g_{i}\left(R_{a_{1}}, \ldots, R_{a_{r}}, L_{a_{1}}, \ldots, L_{a_{r}}\right)=0$, where the $g_{i}$ are the birrepresentation defining identities of the variety $\mathcal{V}$. Then $\mathcal{M}(A)$ is a multiplicative universal envelope for $A$. In the case of our example the relations are:

$$
\begin{gathered}
R_{x}-L_{x}=0 \\
R_{(y x) x}+R_{x} R_{y x}+R_{x}^{2} R_{y}+\gamma\left[R_{y} R_{x^{2}}+2 R_{y} R_{x}^{2}\right]=0 \\
R_{x}^{3}+\gamma R_{x^{3}}=0 .
\end{gathered}
$$

So, our goal is to prove that this algebra $\mathcal{M}(A)$ is nilpotent and therefore finite-dimensional when ever $A$ is finitely generated. In other words we want to prove the following

Theorem 2. Let $\mathcal{V}$ be a locally nilpotent variety of algebras and let $A$ be a finitely generated algebra in $\mathcal{V}$. Then the multiplicative universal envelope $\mathcal{M}(A)$ is nilpotent and $\operatorname{dim}(\mathcal{M}(A))<\infty$. 
Proof. As every associative algebra, the multiplicative envelope $\mathcal{M}(A)$ acts faithfully in $M=\mathcal{M}(A) \oplus K 1$, where 1 is a unit element. Therefore, the function $(R, L): A \longrightarrow \mathcal{M}(A) \times \mathcal{M}(A) \subseteq \operatorname{End}(M) \times \operatorname{End}(M)$ becomes a birrepresentation of $A$ in the vector space $M$. Since $M$ is generated as an $A$-bimodule by the set $\left\{R_{a}, L_{a} \mid a \in A\right\} \cup\{1\}$ and $A$ is finitely generated lemma 2 implies that the split null extension $S=A \oplus M$ is finitely generated. Since $R$ and $L$ satisfies the defining identities $g_{i}$ we have that $S$ belogs to the variety $\mathcal{V}$. We conclude that $S$ is nilpotent. Since $\mathcal{M}(A)=B=\left\langle R_{x}, L_{x} \mid x \in A\right\rangle$, corollary 1 implies that $\mathcal{M}(A)$ is nilpotent. Finally we deduce from remark 2 that $\mathcal{M}(A)$ is finite-dimensional.

\title{
Acknowledgement
}

The authors thank the referee for his (her)comments that improved the presentation of the paper.

\section{References}

[BEL] A. Behn, A. Elduque, A. Labra, A class of Locally Nilpotent Commutative Algebras, International Journal of Algebra and Computation, 21, No. 5, pp. 763 - 774, (2011).

[CHL] I. Correa, I. R. Hentzel, A. Labra, Nilpotency of Commutative Finitely Generated Algebras Satisfying $L_{x}^{3}+\gamma L_{x^{3}}=0, \gamma=1,0$ Journal of Algebra 330, pp. 48-59, (2011).

[Eil] S. Eilenberg, Extensions of general algebras. Ann. Soc. Polon. Math. 21, pp. 125-134, (1948).

[Um] U. Umirbaev, Universal enveloping algebras and derivations of Poisson algebras. Arxiv. 1102 0366v 2 feb. 2011.

\author{
Manuel Arenas \\ Departamento de Matemáticas, \\ Facultad de Ciencias, \\ Universidad de Chile \\ Casilla 653, Santiago, \\ Chile \\ e-mail : mcarenas@yahoo.com \\ and
}




\author{
Alicia Labra \\ Departamento de Matemáticas, \\ Facultad de Ciencias, \\ Universidad de Chile \\ Casilla 653, Santiago, \\ Chile \\ e-mail : alimat@uchile.cl
}

